\title{
VT-Based Phase Envelope and Flash Calculations in the Presence of Capillary Pressure
}

\author{
Sandoval, Diego R.; Michelsen, Michael L.; Yan, Wei; Stenby, Erling Halfdan
}

Published in:

Industrial and Engineering Chemistry Research

Link to article, DOI:

10.1021/acs.iecr.8b05976

Publication date:

2019

Document Version

Peer reviewed version

Link back to DTU Orbit

Citation (APA):

Sandoval, D. R., Michelsen, M. L., Yan, W., \& Stenby, E. H. (2019). VT-Based Phase Envelope and Flash Calculations in the Presence of Capillary Pressure. Industrial and Engineering Chemistry Research, 58(13), 5291-5300. https://doi.org/10.1021/acs.iecr.8b05976

\section{General rights}

Copyright and moral rights for the publications made accessible in the public portal are retained by the authors and/or other copyright owners and it is a condition of accessing publications that users recognise and abide by the legal requirements associated with these rights.

- Users may download and print one copy of any publication from the public portal for the purpose of private study or research.

- You may not further distribute the material or use it for any profit-making activity or commercial gain

- You may freely distribute the URL identifying the publication in the public portal 


\title{
VT-based Phase Envelope and Flash Calculations Including Capillary Pressure
}

\author{
Diego R. Sandoval, ${ }^{\dagger}$ Michael L. Michelsen, ${ }^{\ddagger}$ Wei Yan, ${ }^{* \dagger}$ and Erling H. Stenby ${ }^{\dagger}$ \\ $\dagger$ Center for Energy Resources Engineering (CERE), Department of Chemistry, Technical \\ University of Denmark, Lyngby 2800 \\ $\ddagger$ Center for Energy Resources Engineering (CERE), Department of Chemical Engineering, \\ Technical University of Denmark, Lyngby 2800 \\ E-mail: *weya@kemi.dtu.dk
}

\begin{abstract}
Phase envelope construction and isothermal flash are two widely used phase equilibrium calculations. Because of the increasing importance of oil and gas production from shale, there is a need to perform these calculations in the presence of capillary pressure. Such calculations are also important for other processes inside porous media. Classical phase equilibrium calculations usually use $P T$-based thermodynamics that requires the solution of the equation of state (EoS) for the molar volume or density to calculate the desired thermodynamic properties. An alternative to this formulation is VT-based thermodynamics where repeated molar volume or density solutions are avoided by co-solving the equality of phase pressures in the equilibrium calculation. The formulation is particularly advantageous for equilibrium calculation with capillary pressure. It can naturally handle the negative pressures in the wetting phase (usually the liquid) caused by the capillary pressure. Furthermore, the capillary pressure function usually has a high level of implicitness for the pressure variable which can be circumvented in the $V T$-formulation. We present the $V T$-based formulations for
\end{abstract}


phase envelope construction and isothermal flash with capillary pressure. The incorporation of capillary pressure actually does not increase the number of equations since the pressure equality equation is included in the VT-based formulations without capillary pressure. Using volume as an independent variable result in simpler derivatives for the interfacial tension models, which is more suitable for cases with large capillary pressures or with saturation dependent capillary pressures that account for pore size distribution. The developed algorithms are tested for multicomponent reservoir fluid examples, including natural gas, gas condensate, and black oil, down to extremely small pore sizes. The algorithms converge with only a few Newton iterations even for

the extreme cases. For the cases where pore size distribution is considered and the capillary pressure is treated as saturation dependent, the same convergence behavior is kept. This feature is especially attractive for reservoir simulation cases in which saturation dependent capillary pressure curves are used.

\section{Introduction}

Phase equilibrium calculation is of utter importance in various applications from a simple separator or storage tank design to whole process simulation in the petroleum or other industries. Phase equilibrium problems are usually solved using the so-called pressure based thermodynamics, where pressure, together with temperature and mole numbers, are selected as the independent variables in the calculation of thermodynamic properties. This approach is abbreviated as PT-based thermodynamics although other abbreviations like $P$-based or NPT based are also common in the literature. The choice is considered as convenient since pressure, similar to temperature, is easy to measure and often a variable used in the problem specification. However, most equations of state (EoS) are formulated as explicit functions of volume, temperature, and mole numbers. When we evaluate thermodynamic properties such as fugacity coefficients using these EoS at a given pressure, it is necessary to solve for the volume first. It means that at the innermost layer of thermodynamic properties 
calculation we always need to use volume as an independent variable even if $P T$-based thermodynamics is used. For simple cubic EoS solving for the volume is easy and efficient. However, for complex EoS, especially the SAFT-type ones having an association term, the volume or density solution is more expensive and should be avoided if possible. Hence, for the mathematical formulation using $P$-explicit EoS models, it appears a more natural choice to use volume as an independent variable instead of pressure. The resulting approach is termed as $V T$-based thermodynamics.

$V T$-based thermodynamics has been used in various phase equilibrium calculations. The critical point calculation algorithm proposed by Heidemann and Khalil ${ }^{1}$ is one of the first attempts using VT-based thermodynamics. Nagarajan et al. ${ }^{2,3}$ suggested using independent variables other than the PT-based ones in stability testing, flash calculation and critical point determination. They proposed to use molar densities as independent variables to obtain smooth functions and to avoid possible discontinuities when using mole fractions and molar volumes. Michelsen ${ }^{4}$ generalized the solution of the flash problem to six thermodynamic state function based flash specifications ( $P T, P H, P S, T V, U V$ and $S V$ ) using either PT-based or VT-based thermodynamics. He pointed out that both VT flash and PT flash can be formulated as unconstrained minimization of a thermodynamic state function when using VT-based thermodynamics. This is another attractive feature for $V T$-based thermodynamics since in contrast only $P T$ flash is an unconstrained minimization among the six flash problems if PT-based thermodynamics is used. Recent years have seen many studies $^{5-10}$ on stability analysis and phase split calculation of the isochoric problem since VT flash is by nature an unconstrained minimization problem using VT-based thermodynamics. For a different application, Halldòrsson and Stenby ${ }^{11}$ presented a $V T$-based formulation to calculate isothermal gravitational segregation. The solution exhibited a better behavior than the PT-based formulation by giving a smoother convergence. Pereira et al. ${ }^{12}$ presented a comparison of the computational speed between PT-based and VT-based thermodynamics for PT-flash using a SAFT-type EoS. The obtained results indicate a better performance of 
the VT-based formulation especially when the EoS becomes more complex and the number of components increases. Recently, Paterson et al. ${ }^{13}$ presented new RAND-based formulations for multiple phases and chemical equilibrium calculations as an extension of the original ideal RAND formulation by White et al. ${ }^{14}$ Both the PT-based modified-RAND and the VTbased vol-RAND were presented. Both formulations were compared using different EoS and systems with a different number of phases. The superiority of vol-RAND over the modified RAND is more noticeable as the complexity of the EoS increases. Paterson et al. ${ }^{15}$ further evaluated the performance of the VT-based formulation (not RAND formulation) in slimtube and reservoir simulations, showing again improvements over the $P T$-based formulation for complex SAFT-type EoS.

Apart from the various existing applications of $V T$-based thermodynamics, some other equilibrium calculation problems can also benefit from the approach. One such problem is equilibrium calculation involving capillary pressure effects, which has been investigated using PT-based thermodynamics. ${ }^{16-19}$ One of the main advantages of using VT-based thermodynamics is that most of the interfacial tension models are explicit functions of $V, T$ and $n$, and the derivatives w.r.t. $V$ are simpler and more straightforward than w.r.t. $P$. In addition, pore size dependent capillary pressure is usually formulated as saturation (volume fraction) dependent. The dependence is easier to handle using VT-based thermodynamics due to the direct relation between saturation and phase volumes. Another advantage of $V T$-based thermodynamics, in the presence or absence of capillary pressure, is that negative pressure values can be easily handled as compared with PT-based thermodynamics. Finally, it can be shown that $V T$-based phase envelope or flash calculation has the same number of equations as the corresponding $P T$-based one in the presence of capillary pressure.

Phase equilibria calculation under capillary pressure effects has gained more attention in the last decade due to the increasing interest in developing shale and tight reservoirs. Phase equilibrium in extremely tight pores can deviate from the conventional bulk fluid behavior due to fluid-wall interactions and geometry confinement. Fluid-wall interactions are respon- 
sible for fluid adsorption close to the wall and for the capillary pressure arising from the curved interfaces bridging from one wall to another as a continuum media. The assumption of continuous fluid and interface can be questioned for pores down to nanometer scales, nevertheless, the Kelvin equation has been validated experimentally for pure hydrocarbon systems down to a few nanometers. Fisher and Israelachvili ${ }^{20}$ observed capillary condensation of cyclohexane between crossed mica cylinders at different radii $\left(r_{c} \geq 4 \mathrm{~nm}\right)$. Recently, direct observation ${ }^{21,22}$ of propane and n-butane capillary condensation using nanofluidic devices was reported down to $8 \mathrm{~nm}$ pore sizes $\left(r_{c} \approx 4 \mathrm{~nm}\right)$. The observed deviations of the Kelvin equation are typically under $5 \%$. For pore size analysis lower than $8 \mathrm{~nm}$, the Kelvin equation can introduce higher deviations and DFT-type methods are recommended. ${ }^{23}$ For the multicomponent case, experimental measurements are more challenging due to diffusion effects and accumulation of components at the interface. Interpretation of the experimental results becomes difficult and a direct quantitative validation of theories such as the non-ideal multicomponent Kelvin equation ${ }^{24}$ is not possible. Luo et al. ${ }^{25}$ measured the bubble point of binary hydrocarbon mixtures using calorimetry techniques. A suppression of the bubble point was observed for all the cases. Wang et al. ${ }^{26}$ and Alfi et al. ${ }^{27}$ measured the bubble point of hydrocarbon mixtures using nanofluidic devices, observing as well a decrease in the bubble point.

For extremely small pore sizes that can hold just a few molecules, the continuous medium assumption is questionable and the geometrical restrictions of the molecules inside the pore should be taken into account. Besides the expected change in density due to molecular redistribution, a less intuitive consequence is the shift of the critical properties. Several researchers have tried to address this observation by an artificial modification of the critical parameters in cubic EoS. This approach is however over simplistic and lacks a sound theoretical basis. A more appropriate way is to explicitly consider the wall potential and geometry in the final model, such as the EoS developed by Travalloni et al. ${ }^{28}$ Some descriptive adsorption models like the multicomponent theory of adsorption (MPTA) ${ }^{29}$ or the simplified local 
density theory $(\mathrm{SLD})^{30}$ have essentially considered the wall potential, and should somewhat capture the strong confinement effects.

In our current study, we limit our discussion to the range where capillary pressure effects described by the Young-Laplace equation are considered to be the major effect. The other effects including adsorption and geometrical confinement are neglected. We focus on the algorithmic aspect of phase equilibrium calculation including capillary pressure. We construct phase envelope and flash calculation in the presence of capillary pressure using $V T$-based thermodynamics. In the presented examples, we do have cases with capillary radii smaller than $4 \mathrm{~nm}$. They are mainly for illustrative purposes, i.e. to examine the reliability of the developed algorithm. The actual physical effects of capillary pressure at very small pore radii (e.g. below $4 \mathrm{~nm}$ ) should be discussed with caution.

In the remainder of the article, we first present the methods for $V T$-based phase envelope construction and VT-based flash, both with and without capillary pressure. We then present results for phase envelope and flash calculation, respectively, for cases with capillary pressure.

\section{Methods}

A VT-based thermodynamic formulation differs from the classical PT-based one in that the system properties and their derivatives are obtained at specified $(V, T, \boldsymbol{n})$ instead of $(P, T, \boldsymbol{n})$. A VT-based thermodynamic module can be developed through a slight modification of a PTbased thermodynamic module. In a $P T$-based module, fugacity coefficients $\varphi_{i}$ are calculated by

$$
R T \ln \varphi_{i}(\boldsymbol{n}, P, T)=-\frac{\partial}{\partial n_{i}}\left[\int_{\infty}^{V}\left(P-\frac{n R T}{V}\right) \mathrm{d} V\right]_{V, T, n_{j \neq i}}-R T \ln Z
$$

where $R$ is the universal gas constant, $Z$ is the compressibility factor, and $\boldsymbol{n}$ the mole numbers vector. In the $V T$-based formulation, $\ln P$ is added on both sides of (Eq. 1), resulting in

$$
R T \ln F_{i}(\boldsymbol{n}, V, T)=-\frac{\partial}{\partial n_{i}}\left[\int_{\infty}^{V}\left(P-\frac{n R T}{V}\right) \mathrm{d} V\right]_{V, T, n_{j \neq i}}-R T \ln \frac{V}{n R T}
$$


where $F_{i}$ is defined as $P \varphi_{i}$ or $f_{i} / z_{i}$. The reformulation of (Eq. 1) to (Eq. 2), and the introduction of $F_{i}^{18,31,32}$ avoid logarithms of negative compressibility factors and negative fugacity coefficients, respectively. Notice that for pure component calculations $F=f$.

\section{Capillary Pressure Models}

Integration of the capillary pressure into phase equilibrium problems using $V T$-based formulation may be advantageous over the $P T$-based formulation. Capillary pressure is usually expressed as a function of interfacial tension and pore radius. For interfacial tension, we use the parachor model presented by McLeod and Sugden ${ }^{33}$ where the interfacial tension $\sigma$ is a relatively simple expression in $V$ and $\boldsymbol{n}$,

$$
\sigma=\left[\sum_{i}^{N_{c}} \frac{\prod_{i} n_{i}^{l}}{V^{l}}-\sum_{i}^{N_{c}} \frac{\prod_{i} n_{i}^{g}}{V^{g}}\right]^{E}
$$

where $N_{c}$ is the number of components, $E$ is the scaling exponent and $\Pi$ is the parachor value. The saturation dependent functions on the capillary pressure can be easily incorporated due to the direct relationship between saturation and the phase volumes. This means that pore size distribution of heterogeneous porous media can be easily considered in the problem formulation. A general expression for the capillary pressure inside porous media can be written as the Young-Laplace equation inside a capillary tube with a variable radius.

$$
P_{c}=\frac{2 \sigma \cos \theta}{r_{c}(S)}, \quad \text { with } \quad S=\frac{V^{l}}{V^{g}+V^{l}}
$$

where $S$ is the liquid saturation, $\theta$ is the contact angle between the wetting phase and the wall, and $r_{c}(S)$ is the effective capillary radius at a given saturation. An example of a saturation dependent function is the widely used Leverett J-function in petroleum engineering problems. The function relates the capillary pressure to the porosity $\phi$, rock permeability $k$, 
and saturation $S$

$$
J(S)=\frac{P_{c}(S) \sqrt{k / \phi}}{\sigma \cos \theta}
$$

If we replace (Eq. 4) in (Eq. 5) we obtain an explicit expression for the effective capillary radius as a function of the saturation

$$
r(S)=\frac{2 \sqrt{k / \phi}}{J(S)}
$$

\section{Construction of Phase Envelope with Capillary Pressure}

A single saturation point calculation is a phase equilibrium problem in which a phase fraction

$\beta$ is set to be 0 or 1 and an additional condition of the system is given. Such a condition is generally a specification of one of the intensive properties of the system. For instance, the specification of the temperature:

$$
S_{1}(T)=T-T_{\text {spec }}=0
$$

It is not unusual to have convergence problems when the conditions are close to the critical point, cricondenbar, or cricondentherm where close initial estimates are required. Therefore, it is recommended to construct the phase envelope in a sequential manner using previous points to generate initial estimates of the current calculation, rather than a single-blind saturation point calculation. ${ }^{34,35}$

A system at equilibrium implies that the system is at thermal, mechanical, and chemical equilibrium. This means that the temperature and pressure are uniform throughout the system, and the chemical potentials of each component are the same in all the phases. Standard phase equilibrium calculations using pressure and temperature as independent variables satisfy the thermal and mechanical equilibrium implicitly since the properties of all the phases are calculated at the same temperature and pressure. As a consequence, only the chemical equilibrium conditions need to be taken care of. When VT-based thermodynamics 
is used, the volume of each phase is used as an independent variable instead of the system pressure. Consequently, the phase pressures are not necessarily equal during iterations but only at the solution (i.e. equilibrium). Therefore, an additional equation for the pressure equilibrium in the system is required.

Our VT-based phase envelope algorithm with capillary pressure is a direct extension of Michelsen's VT-based phase envelope algorithm without capillary pressure. ${ }^{31}$ Michelsen's $V T$-phase envelope code is developed by slightly modifying his PT-based phase envelope algorithm. ${ }^{34}$ The $V T$-based algorithm can however trace the envelope in the region of negative pressures easily and save the time used in repeated density solution for complex EoS. Although Kunz et al. ${ }^{31}$ have given a description of Michelsen's code, we briefly describe Michelsen's VT-based algorithm without capillary pressure here for the completeness. The $V T$-based phase envelope algorithm is based on the following formulation:

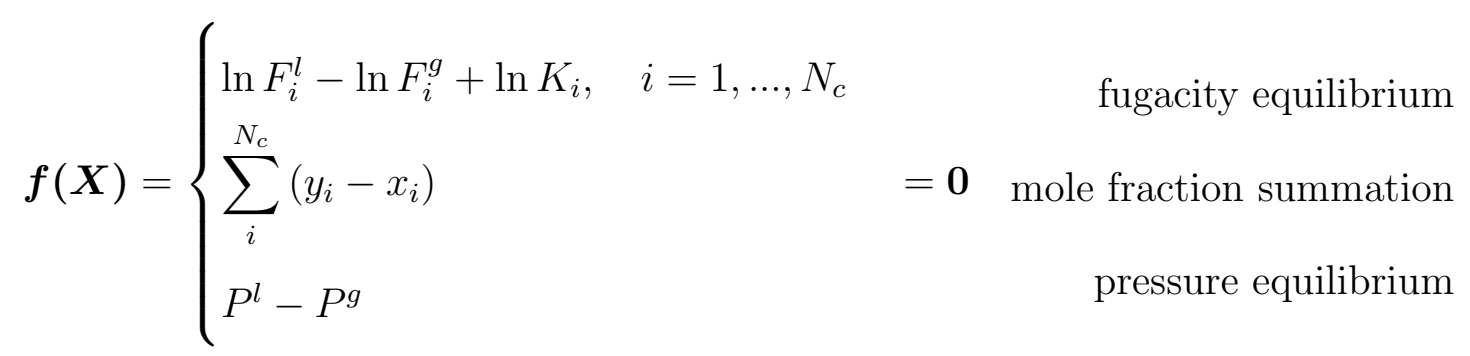

where $x_{i}$ and $y_{i}$ the mole fraction of the liquid and gas phase respectively, $P^{l}$ and $P^{g}$ the pressure in the liquid and gas phases. The equilibrium factors $K_{i}$ are defined as the ratio between the mole fractions

$$
K_{i}=\frac{y_{i}}{x_{i}}
$$

The mass balance follows that

$$
x_{i}=\frac{z_{i}}{1-\beta+\beta K_{i}}, \quad y_{i}=\frac{z_{i} K_{i}}{1-\beta+\beta K_{i}}
$$


where $\beta$ is the molar vapor fraction. The independent variables are,

$$
X=\left\{\ln K_{i}, \ln T, \ln v^{l}, \ln v^{g}\right\}
$$

where, $T$ is the temperature, $v^{l}$ and $v^{g}$ are the molar volumes of the liquid phase and gas phase respectively. Here we use the molar volumes instead of total phase volumes as independent variables. Use of molar volumes is actually equivalent to use of phase volumes because at a fixed $\beta$, the phase volumes are proportional to the respective phase molar volumes. However, the use of molar volumes avoids the situation where phase volumes become zero (for $\beta=0$ or 1) and their logarithms become indefinite.

There are $N_{c}+3$ variables but $N_{c}+2$ equations in (Eq. 8). One additional equation is needed and it is called here the 'specification equation'. The specification equation is chosen by the user and specifies the value of one of the independent variables to be fixed during the saturation point calculation. The result of the new set of equations is as follows:

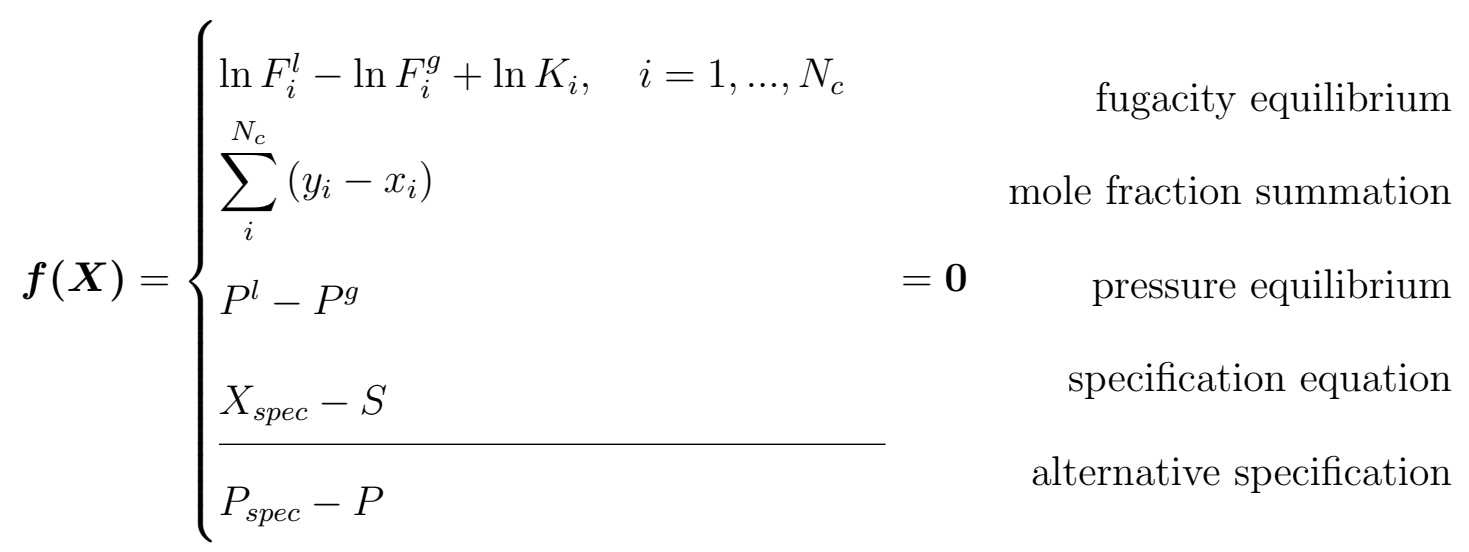

where $X_{\text {spec }}$ the specification variable, and $S$ the specified value of $X_{\text {spec }}$. For instance, if $X_{\text {spec }}$ is $\ln T, S$ is the logarithmic value of the temperature at which a saturation point calculation is desired. It should be noted that we have added in (Eq. 11) an alternative specification equation for $P$. This additional equation allows more flexibility and we can use $P$ as the specification variable if needed.

The effect of the capillary pressure can be added by modifying the pressure equilibrium 
equation in the system of equations in (Eq. 8). The resulting system of equations is the following

$$
\boldsymbol{f}(\boldsymbol{X})=\left\{\begin{array}{lr}
\ln F_{i}^{l}-\ln F_{i}^{g}+\ln K_{i}, \quad i=1, \ldots, N_{c} & \text { fugacity equilibrium } \\
\sum_{i}^{N_{c}}\left(y_{i}-x_{i}\right) & =\mathbf{0} \\
P^{l}-P^{g}+P_{c}\left(\boldsymbol{x}, \boldsymbol{y}, v^{l}, v^{g}\right) & \text { mole fraction summation } \\
X_{\text {spec }}-S & \text { specification equation }
\end{array}\right.
$$

where the independent variables are kept the same as (Eq. 10), and the specification equation has he same meaning as in (Eq. 11). In comparison with the previous formulation presented by Sandoval et al., ${ }^{18}$ the number of equations remains the same after adding the capillary pressure effect. Moreover, if the simple parachor model for the interfacial tension in (Eq. 3) is used, the derivatives of the capillary pressure are readily obtained due to the explicit form with respect the mole fractions and molar volumes.

$$
\begin{aligned}
\frac{\partial \sigma}{\partial \ln K_{i}} & =\left((1-\beta) \frac{\partial \sigma}{\partial y_{i}}-\beta \frac{\partial \sigma}{\partial x_{i}}\right) \frac{x_{i} y_{i}}{z_{i}} \\
\frac{\partial \sigma}{\partial \ln v^{g}} & =\frac{\xi}{v^{g}} \sum_{i}^{N_{c}} \Pi_{i} y_{i} \\
\frac{\partial \sigma}{\partial \ln v^{l}} & =-\frac{\xi}{v^{l}} \sum_{i}^{N_{c}} \Pi_{i} x_{i}
\end{aligned}
$$

where

$$
\xi=E \sigma^{\frac{E-1}{E}}, \quad \frac{\partial \sigma}{\partial y_{i}}=-\xi \frac{\Pi_{i}}{v^{g}}, \quad \frac{\partial \sigma}{\partial y_{i}}=-\xi \frac{\Pi_{i}}{v^{l}}
$$

\section{Initialization and Sequential Construction}

The initialization is made at a low pressure using traditional PT-based thermodynamics. The calculation is started at a specified phase fraction $\beta=1,0$ for the phase envelope, and $0<\beta<1$ for the quality lines. Wilson's approximation is used to initialize the equilibrium 
$K$-factors. It is then followed by 3-5 iterations using a partial Newton iteration method neglecting the composition derivatives. The procedure is switched to a full Newton step scheme using the set of independent variables $\boldsymbol{X}$ from (Eq. 10). When switching to the $V T$-based variables, the volume of each phase is calculated from the EoS and one is selected for the specification equation at the first iteration. The strategy presented in Michelsen ${ }^{34}$ can be employed to sequentially construct the phase envelope using sensitivity analysis

$$
\frac{\partial \boldsymbol{f}}{\partial \boldsymbol{X}} \frac{\partial \boldsymbol{X}}{\partial S}+\frac{\partial \boldsymbol{f}}{\partial S}=\mathbf{0}
$$

where $(\partial \boldsymbol{X} / \partial S)$ is the vector of sensitivities. The most sensitive variable is used to specify the next calculation point, and $(\partial \boldsymbol{X} / \partial S)$ is used to generate the initial estimates.

For the formulation in Eq. (11), the alternative specification can also be included in the sensitivity analysis. In such case, the Jacobian $(\partial \boldsymbol{f} / \partial \boldsymbol{X})$ should be constructed for the system with the alternative equation, and $P$ is treated as an additional element in the vector $\boldsymbol{X}$. Moreover, to have a fair comparison with the other variables, the sensitivity of $P$ should be calculated in a 'logarithmic' manner as follows:

$$
\frac{\partial \log P}{\partial S} \approx \frac{1}{1+|P|} \frac{\partial P}{\partial S}
$$

where the denominator has been modified to avoid indefinite values. Having the sensitivity of $P$ allows construction of a 3rd degree polynomial for interpolating/extrapolating to a specified pressure value. A flowchart is provided in supporting information for visualization of the described procedure.

\section{The PT-flash using VT-based Thermodynamics}

The solution of the PT-flash problem using PT-based thermodynamics corresponds to the minimum of the system Gibbs energy. The problem is usually formulated as a minimization 
subject to linear constraints on the mole numbers:

$$
\min _{\boldsymbol{n}^{\boldsymbol{g}}, \boldsymbol{n}^{l}} G\left(T_{\text {spec }}, P_{\text {spec }}, \boldsymbol{n}^{\boldsymbol{g}}, \boldsymbol{n}^{\boldsymbol{l}}\right), \quad \text { s.t. } \quad \boldsymbol{n}^{\boldsymbol{z}}=\boldsymbol{n}^{\boldsymbol{g}}+\boldsymbol{n}^{\boldsymbol{l}}
$$

By setting the mole numbers in a certain phase (e.g. the liquid phase) as dependent $n_{i}^{l}=n_{i}^{z}-n_{i}^{g}$, we convert the original problem to an unconstrained minimization with $N_{c}$ variables:

$$
\min _{\boldsymbol{n}^{\boldsymbol{g}}} G\left(T_{\text {spec }}, P_{\text {spec }}, \boldsymbol{n}^{\boldsymbol{g}}\right)
$$

Michelsen ${ }^{4}$ showed that PT-flash can also be formulated as an unconstrained minimization problem when $V T$-based thermodynamics is used. The state function to be minimized with respect the mole numbers and the volume is

$$
\min _{\boldsymbol{n}^{\boldsymbol{g}}, V^{l}, V^{g}} Q=\min _{\mathbf{n}^{\mathbf{g}}, V^{l}, V^{g}}\left\{A\left(T_{\text {spec }}, \boldsymbol{n}^{\boldsymbol{g}}, V^{l}, V^{g}\right)+V P_{\text {spec }}\right\}
$$

where $A$ is the Helmholtz energy and $V=V^{l}+V^{g}$. The fact that the problem remains a minimization problem for $V T$-based thermodynamics is particularly attractive since standard minimization procedures using Newton steps can be employed

$$
\Delta x=-H^{-1} g
$$

where $\boldsymbol{x}$ is the set of independent variables $\boldsymbol{x}=\left\{\boldsymbol{n}^{g}, V^{g}, V^{l}\right\}$. The gradient of the system has the following form

$$
\boldsymbol{g}= \begin{cases}g_{i} & =\frac{\partial \tilde{Q}}{\partial n_{i}^{g}}=\ln f_{i}^{g}-\ln f_{i}^{l}, \quad i=1, \ldots, N_{c} \\ g_{N_{c}+1} & =\frac{\partial \tilde{Q}}{\partial V^{g}}=\frac{1}{R T}\left(-P^{g}+P_{\text {spec }}\right) \\ g_{N_{c}+2} & =\frac{\partial \tilde{Q}}{\partial V^{l}}=\frac{1}{R T}\left(-P^{l}+P_{\text {spec }}\right)\end{cases}
$$

where $\tilde{Q}=Q / R T$ has been introduced for convenience. The Hessian matrix can be written 
as follows

$$
=\left\{\begin{array}{l}
H_{i j}=\frac{\partial g_{i}}{\partial n_{j}^{g}}=\frac{\partial \ln F_{i}^{g}}{\partial n_{j}^{g}}-\frac{1}{\beta^{g}}+\frac{\delta_{i j}}{V^{g}}+\frac{\partial \ln F_{i}^{l}}{\partial n_{j}^{l}}-\frac{1}{\beta^{l}}+\frac{\delta_{i j}}{V^{l}}, \quad i, j=1, \ldots, N_{c} \\
H_{i, N_{c}+1}=\frac{\partial \ln F_{i}^{g}}{\partial V^{l}} \\
H_{i, N_{c}+2}=\frac{\partial g_{i}}{\partial V^{l}}=-\frac{\partial \ln F_{i}^{g}}{\partial V^{l}} \\
H_{N_{c}+1, j}=\frac{\partial g_{N_{c}+1}}{\partial n_{j}^{g}}=-\frac{1}{R T} \frac{\partial P^{g}}{\partial n_{j}^{g}}=\frac{\partial \ln F_{i}^{g}}{\partial V^{g}} \\
H_{N_{c}+2, j}=\frac{\partial g_{N_{c}+2}}{\partial n_{j}^{g}}=\frac{1}{R T} \frac{\partial P^{l}}{\partial n_{i}^{l}}=-\frac{\partial \ln F_{i}^{l}}{\partial V^{l}} \\
H_{N_{c}+1, N_{c}+2}=\frac{\partial g_{N_{c}+1}}{\partial V^{l}}=0 \\
H_{N_{c}+2, N_{c}+1}=\frac{\partial g_{N_{c}+2}}{\partial V^{g}}=0 \\
H_{N_{c}+1, N_{c}+1}=\frac{\partial g_{N_{c}+1}}{\partial V^{g}}=-\frac{1}{R T} \frac{\partial P^{g}}{\partial V^{g}} \\
H_{N_{c}+2, N_{c}+2}=\frac{\partial g_{N_{c}+2}}{\partial V^{l}}=-\frac{1}{R T} \frac{\partial P^{l}}{\partial V^{l}}
\end{array}\right.
$$

Notice that the elements of the Hessian matrix match those presented by Nichita. ${ }^{36}$ To ensure a decrease of $\tilde{Q}$ at each iteration, control on the step size and corrections to the Hessian may be required to ensure positive definiteness. ${ }^{37,38}$ Initialization of the problem can be performed either by stability analysis or by assuming 2-phases using Wilson's approximation for the equilibrium factors. In both cases, one to three updates of the phases fractions and compositions based on PT-based Successive Substitution (i.e. assuming composition independent $K$-factors) are recommended before switching to the $V T$-based Newton step procedure in (Eq. 22).

\section{Inclusion of the Capillary Effect}

Similar to the inclusion of the capillary pressure on the phase envelope calculations presented in the previous section, capillary pressure can be introduced by modifying the gradient in (Eq. 23). The capillary pressure term is included in $g_{N_{c}+1}$ as $+P_{c}$ if the specification pressure 
$P_{\text {spec }}$ is the liquid pressure, or in $g_{N_{c}+2}$ as $-P_{c}$ if $P_{\text {spec }}$ is the gas pressure.

$$
g_{N_{c}+1}=\frac{1}{R T}\left(-P^{g}+P_{\text {spec }}+P_{c}\right) \quad \text { or } \quad g_{N c+2}=\frac{1}{R T}\left(-P^{l}+P_{\text {spec }}-P_{c}\right)
$$

The specified pressure is normally the pressure of the phase that it is taken as reference. Both $P_{l}$ and $P_{g}$ specifications are valid, and the selection of the reference phase is determined by the nature of the problem. For instance, if our interest is to flash a fluid of an oil reservoir, the usual choice of the reference phase is the liquid phase; if our interest is to flash a fluid of a gas condensate, the usual choice of the reference phase is the gas phase. Nevertheless, either choice is allowed in the two cases.

In order to employ a Newton like iteration, modifications to the Hessian are required. The modifications depend on the choice of the reference pressure. When the reference pressure is the liquid pressure, the modifications are the following

$$
P_{s p e c}=P_{l} \rightarrow \begin{cases}H_{N_{c}+1, j} & =\frac{\partial g_{N_{c}+1}}{\partial n_{j}^{g}}=\frac{\partial \ln F_{i}^{g}}{\partial V^{g}}+\frac{1}{R T} \frac{\partial P_{c}}{\partial n^{g}} \\ H_{N_{c}+1, N_{c}+1} & =\frac{\partial g_{N_{c}+1}}{\partial V^{g}}=\frac{1}{R T}\left(-\frac{\partial P^{g}}{\partial V^{g}}+\frac{\partial P_{c}}{\partial V^{g}}\right) \\ H_{N_{c}+1, N_{c}+2} & =\frac{\partial g_{N_{c}+1}}{\partial V^{l}}=\frac{1}{R T} \frac{\partial P_{c}}{\partial V^{l}}\end{cases}
$$

and when the reference pressure is the gas pressure, the modifications are

$$
P_{s p e c}=P_{g} \rightarrow \begin{cases}H_{N_{c}+2, j} & =\frac{\partial g_{N_{c}+1}}{\partial n_{j}^{g}}=-\frac{\partial \ln F_{i}^{l}}{\partial V^{l}}-\frac{1}{R T} \frac{\partial P_{c}}{\partial n^{g}} \\ H_{N_{c}+2, N_{c}+1} & =\frac{\partial g_{N_{c}+1}}{\partial V^{g}}=-\frac{1}{R T} \frac{\partial P_{c}}{\partial V^{g}} \\ H_{N_{c}+2, N_{c}+2} & =\frac{\partial g_{N_{c}+1}}{\partial V^{l}}=-\frac{1}{R T}\left(\frac{\partial P^{g}}{\partial V^{l}}+\frac{\partial P_{c}}{\partial V^{l}}\right)\end{cases}
$$

With these modifications, the Newton update in (Eq. 22) can be employed. It is important to notice that our 'modified Hessian' is formally the Jacobian of the gradient in (Eq. 23) with the modification of (Eq. 25). Although the second order convergence is retained, many 
other properties are lost. Since the Jacobian is no longer symmetric, we cannot use a LDL Cholesky factorization but use a slower LU factorization instead. Moreover, the procedure is no longer a minimization and it is not possible to track the $\mathrm{Q}$ function in (Eq. 21). The problem becomes an equation-solving problem and closer initial estimates are preferred. This may require more successive substitution iterations at the initialization than the bulk flash.

For the 'modified Hessian' capillary pressure derivatives are required. The derivatives of the capillary pressure when using volume as an independent variable are simpler to calculate since most of the interfacial tension models are volume based models. One relevant example is the parachor model in (Eq.3). The necessary derivatives are obtained straightforwardly and have a very simple form with respect to the phase volumes and mole numbers

$$
\begin{aligned}
\frac{\partial \sigma}{\partial n_{j}^{g}} & =-\xi\left(\frac{\Pi_{j}}{V^{l}}+\frac{\Pi_{j}}{V^{g}}\right) \\
\frac{\partial \sigma}{\partial V^{g}} & =\frac{\xi}{V^{g^{2}}} \sum_{i}^{N_{c}} \Pi_{i} n_{i}^{g} \\
\frac{\partial \sigma}{\partial V^{l}} & =-\frac{\xi}{V^{2}} \sum_{i}^{N_{c}} \Pi_{i} n_{i}^{l}
\end{aligned}
$$

The saturation dependent capillary radius in (Eq. 6) can be easily implemented too. The saturation is only a function of the phase volumes and its derivatives are calculated straightforwardly.

$$
\begin{aligned}
\frac{\partial S}{\partial V^{g}} & =-\frac{V^{l}}{\left(V^{g}+V^{l}\right)^{2}} \\
\frac{\partial S}{\partial V^{l}} & =\frac{V^{g}}{\left(V^{g}+V^{l}\right)^{2}}
\end{aligned}
$$

The necessary derivatives to incorporate in (Eq. 26) and (Eq. 27) when using a saturation 
dependent capillary radius are

$$
\begin{aligned}
\frac{\partial P_{c}}{\partial n_{j}^{g}} & =\frac{2 \cos \theta}{r_{c}(S)} \frac{\partial \sigma}{\partial n_{j}^{g}} \\
\frac{\partial P_{c}}{\partial V^{g}} & =\frac{2 \cos \theta}{r_{c}(S)} \frac{\partial \sigma}{\partial V^{g}}-\frac{4 \sigma \cos \theta}{r_{c}(S)^{2}} \frac{\partial r_{c}(S)}{\partial S} \frac{\partial S}{\partial V^{g}} \\
\frac{\partial P_{c}}{\partial V^{l}} & =\frac{2 \cos \theta}{r_{c}(S)} \frac{\partial \sigma}{\partial V^{l}}-\frac{4 \sigma \cos \theta}{r_{c}(S)^{2}} \frac{\partial r_{c}(S)}{\partial S} \frac{\partial S}{\partial V^{l}}
\end{aligned}
$$

Notice that only an additional term in the derivatives of the capillary pressure with respect to the volume is needed when a variable capillary radius is used instead of a fixed one. This property is very attractive and easy to implement in more realistic simulations scenarios where the pore size distribution of the porous media is important.

It is worthwhile mentioning that the presented PT-flash with capillary pressure can be easily extended to the $V T$-flash specification problem. For the original formulation in (Eq. 21), a minimization of the Helmholtz energy $A$ instead of $Q$ is required. The set of independent variables is reduced to from $N_{c}+2$ to $N_{c}+1$ using the linear constraint $V_{g}=V_{\text {spec }}-V_{l}$. As a result, the modified gradient of (Eq. 23) is also reduced to $N_{c}+1$ with $g_{N_{c}+1}=-P_{g}+P_{l}+P_{c}=0$. Solution of the $V T$-flash with capillary pressure using a dynamic model has been recently presented in the literature. ${ }^{39}$

\section{Initialization and Solution Procedure}

Initialization is done using Wilson's equilibrium $K$-factors followed by $P T$-based successive substitution (SS) iterations. The PT-based SS requires solving the EoS but it is more robust than the VT-based SS. For the bulk case, if the first SS iteration yields in an increase of the Gibbs energy or disappearance of a phase occurs, stability analysis is employed. If unstable, a fixed number of SS iterations are taken before switching to Newton like steps. When considering capillary pressure, checks on the Gibbs energy are no longer allowed since it is not a minimization procedure. Therefore, SS iterations are taken until reaching an intermediate tolerance $\approx 0.5$ and the procedure is then switched to a Newton step using 
the Hessian in (Eq. 24) with the modification of (Eq. 26). If a phase disappears during the process, it is confirmed with the modified 'stability analysis' including capillary pressure. ${ }^{40}$

Good initial estimates are required for both the phase split calculation including capillary pressure and the modified stability analysis before switching to second order methods since it is no longer a minimization procedure. Therefore, the procedure is less robust than that of the bulk case.

\section{Results}

\section{Phase Envelope without Capillary Pressure}

The VT-based phase envelope algorithm is applied to System I consisting of a ternary mixture $\mathrm{C}_{1}, \mathrm{C}_{2}$ and $\mathrm{C}_{8}$ taken from Michelsen and Mollerup. ${ }^{35}$ The EoS-parameters are presented in the supporting information. The results for system I are presented in Figure 1. Its construction is started from the dew point branch and continues to the bubble point branch crossing the critical point. The phase envelope has intersecting phase boundaries at the bubble point branch close to $T=200 \mathrm{~K}$. This branch does not represent the real phase boundary since there exists a narrow three-phase region at the vicinity of the intersection which can be confirmed using stability analysis. For further details and discussion of the three-phase region, please refer to Michelsen and Mollerup ${ }^{35}$ on chapter 12 and section 4. Note that the tracing of the phase envelope continues to negative pressures and then comes

back without any problems because $V T$-based thermodynamics can naturally handle negative pressure values. This feature is not immediately obtained in a PT-based formulation. Results with the same features have been obtained by Nichita with his density based phase envelope algorithm. . ${ }^{41}$ 

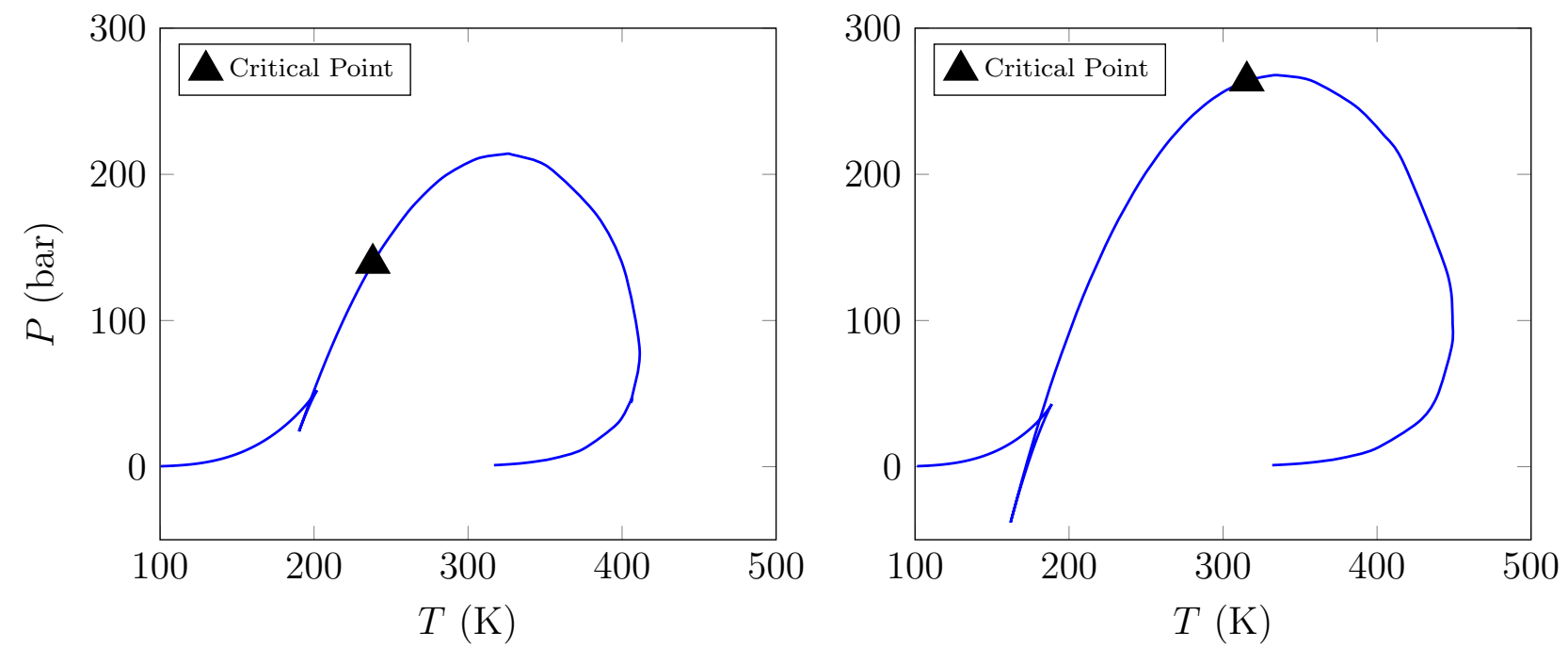

Figure 1: Unusual hydrocarbon ternary system phase envelopes using $V T$-based formulation. $[$ Left $] \boldsymbol{z}_{\text {feed }}=\{0.80,0.15,0.05\} .[$ Right $] \boldsymbol{z}_{\text {feed }}=\{0.85,0.05,0.10\}$

\section{Phase Envelope with Capillary Pressure}

Three examples are presented in this section in order to test the algorithm proposed for the phase envelope. No particular difficulties were observed. The obtained convergence is quadratic as expected due to the Newton-based method approach implemented. For the fluid description, the SRK-EoS was used for System II, III and IV, and the PR(78)-EoS ${ }^{42}$ for System V. The fluid description for each system is given as Supporting Information. The Young-Laplace equation inside a tube in (Eq. 4) is selected to describe the capillary pressure difference between the liquid and gas phase and the parachor model in (Eq. 3) to describe the interfacial tension. The first example in Figure 2 represents a natural gas mixture made up of seven components (System II). The results obtained match those of the PT-based phase envelope calculations including capillary pressure presented by Sandoval et al. ${ }^{18}$ (Figure 2), confirming the correctness of the results presented here. The algorithm handles different capillary radii without any problem, obtaining quadratic convergence in each point along the phase envelope. Shifts along the bubble point and the dew point are observed, except at the critical point. A decrease in the bubble point branch and an inflated dew point branch is observed in all the systems. 
The results for System III and System IV are presented in Figure 3 and Figure 4 and correspond to gas condensate systems described taken from Sherafati and Jessen, ${ }^{40}$ and Whitson and Sunjerga ${ }^{43}$ respectively. The trace amounts of the heaviest components in system III do not seem to affect the stability of the algorithm since the phase envelopes obtained at different capillary radii are completely smooth. A sharp increase in the cricondentherm compared to the other systems is observed. In general, gas condensates show larger shifts in the dew point branch than oil systems. System III shows close to $25 \mathrm{~K}$ shift in the cricondentherm at $5 \mathrm{~nm}$ and $50 \mathrm{~K}$ at $2 \mathrm{~nm}$. System IV shows a more moderate change in the cricondentherm compared to System III. Such difference can be attributed to the heavy components in trace amounts of System III. Furthermore, System IV is a more conventional gas condensate system, and the results are more representative for traditional gas condensate systems. The calculations at $r_{c}=2$ are presented to show the stability of the code at high capillary pressures. However, additional effects such as adsorption and confinement should be taken into account if a more realistic description is required.

System V represents a black oil system from the Eagle Ford field taken from Orangi et al. ${ }^{44}$ Figure 5 shows the results of the phase envelope at different capillary radii. This system is characterized by having large interfacial tension values, yielding capillary pressures above 100 bar for a capillary radius of $2 \mathrm{~nm}$. The system is a special case that represents a black oil heavier than those typically reported for shale reservoirs. However, it exemplifies a fluid in which capillary effects are of huge importance. If the reservoir temperature is between $300-400 \mathrm{~K}$, the saturation pressure shift for a $5 \mathrm{~nm}$ capillary radius is already higher than 20 bar. For the extreme case of $2 \mathrm{~nm}$, the shift of the phase boundary is dramatic. The modified saturation point is shifted to a very low-pressure value below 20 bar. The high capillary pressure values corresponding to this capillary radius show the stability of the code when high negative pressure values are found at low temperatures. 


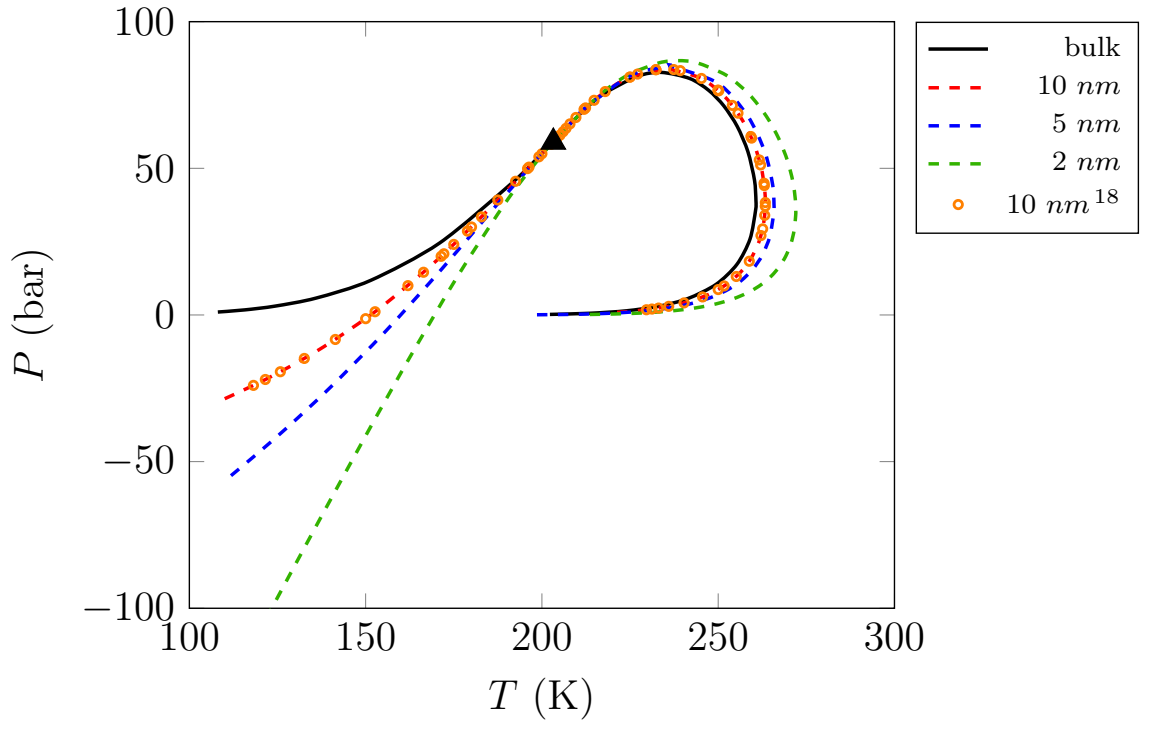

Figure 2: Phase Envelope for a natural gas (system II) at different capillary radii $r_{c}$ including results from ${ }^{18}$ for comparison.

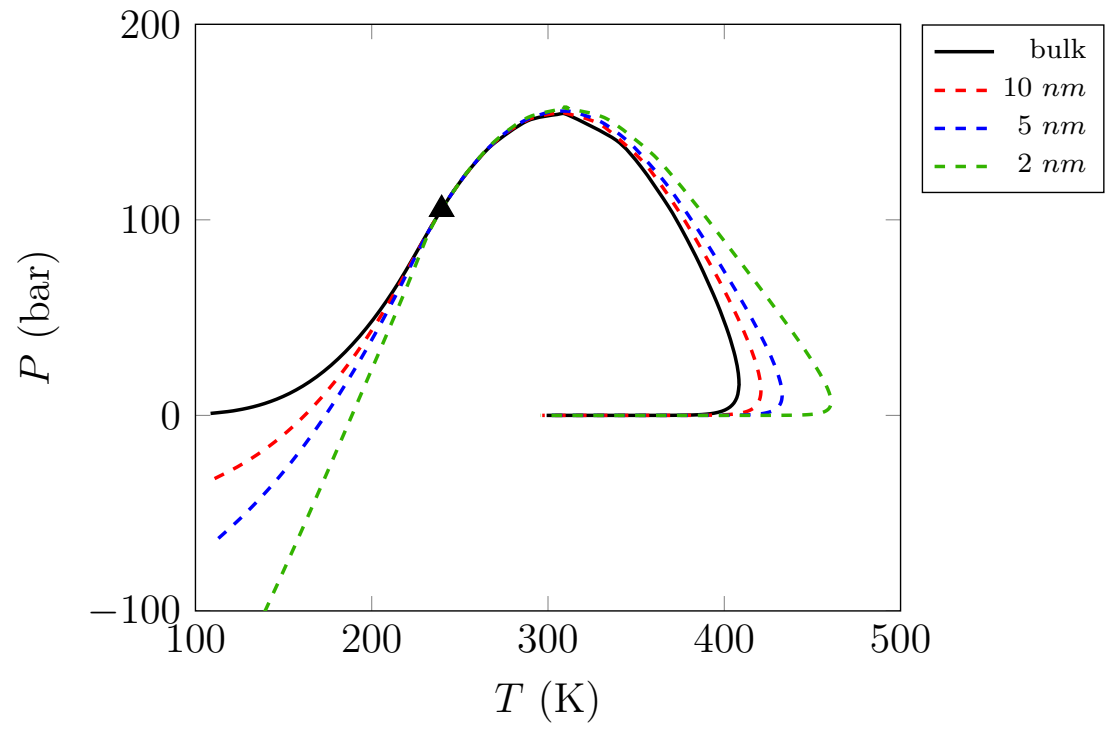

Figure 3: Phase Envelope for a gas condensate (system III) at different capillary radii $r_{c}$ 


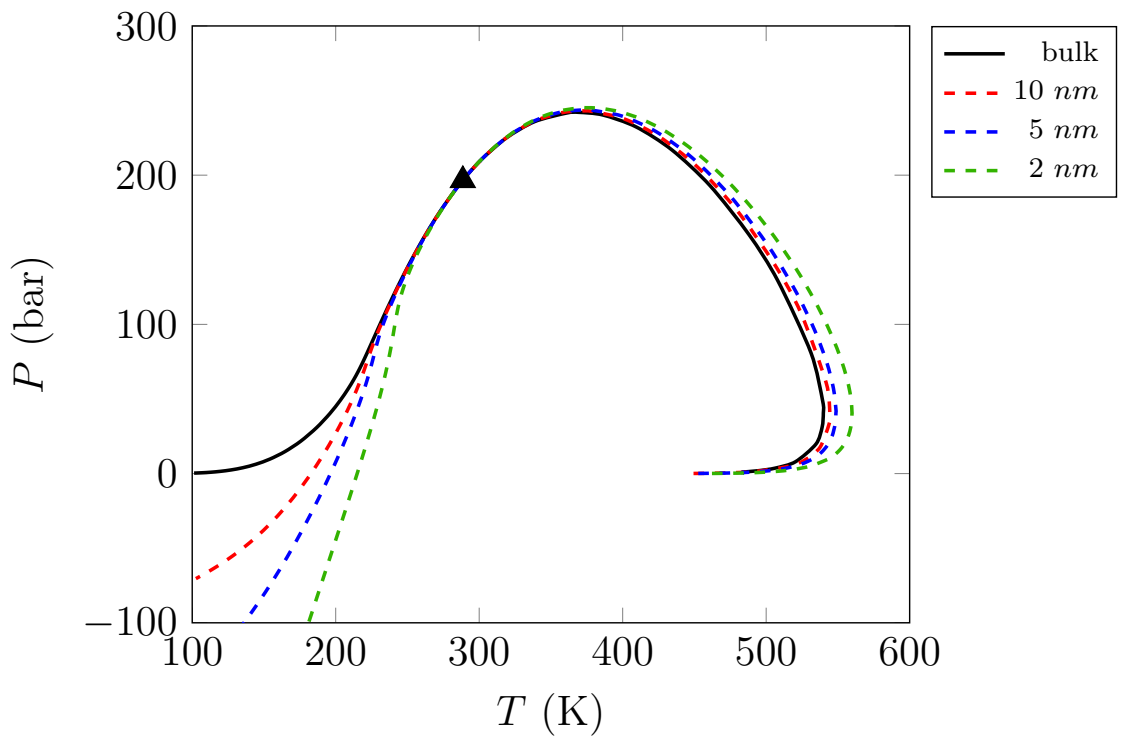

Figure 4: Phase Envelope for a gas condensate (system IV) at different capillary radii $r_{c}$

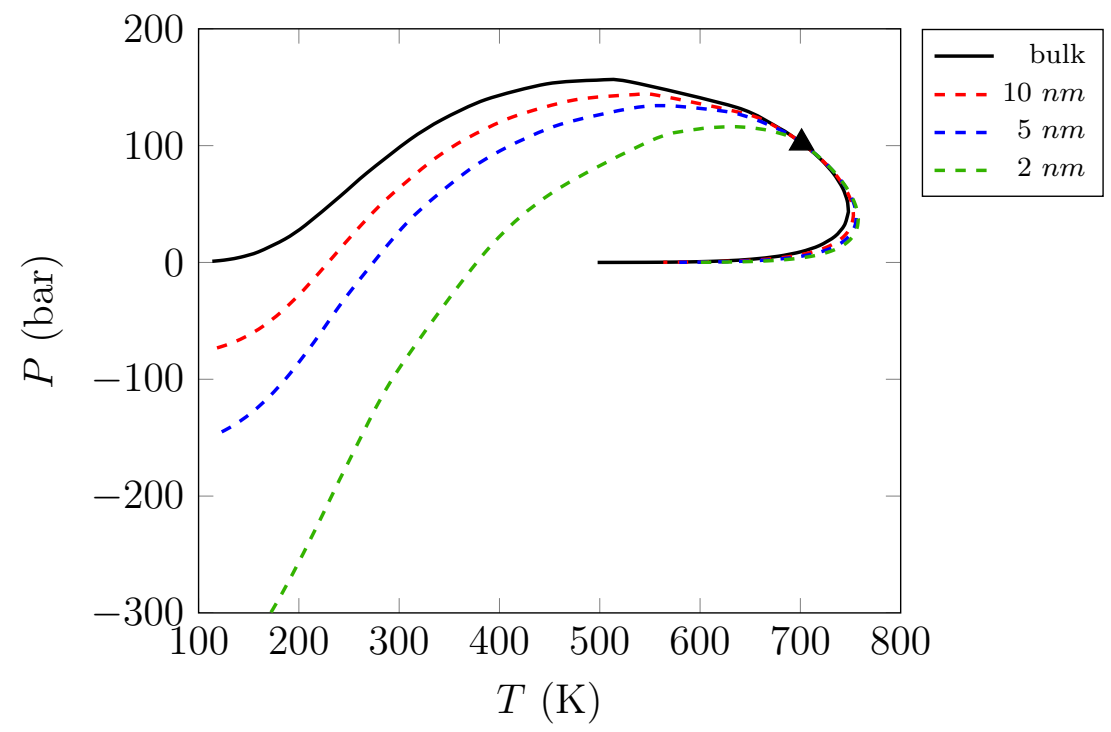

Figure 5: Phase Envelope for Eagle-Ford black oil (system V) at different capillary radii $r_{c}$ 


\section{Flash}

The flash code was tested for system II, III, IV, and V at different liquid pressure and temperature specifications. The presented examples show the results at different capillary radii including an example of a saturation dependent capillary radius shown in Figure 6. The rock permeability and rock porosity in (Eq. 6) were taken in such a way that the range of $r_{c}(S)$ is within the same range of the constant capillary radii used for different examples. The comparison in this way makes more sense. The flash algorithm was tested with the same systems used for the phase envelope. The algorithm shows quadratic convergence for all the cases, including the case using saturation dependent radii. The iteration count until reaching a tight convergence tolerance are presented in Table 1, Table 2, Table 3, and Table 4. The number of iterations is comparable within all examples, with more iterations as the capillary radius decreases.
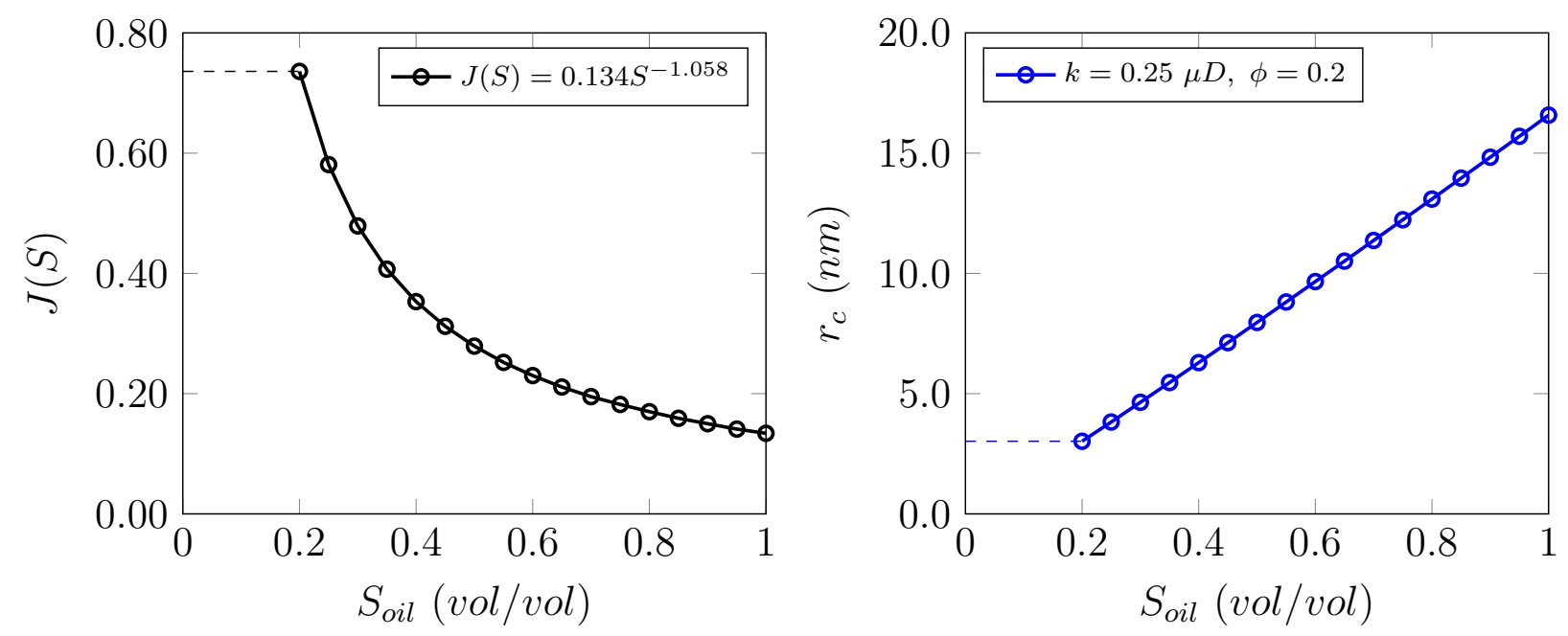

Figure 6: [Left] J-function taken from Brooks and Corey. ${ }^{45}$ [Right $]$ Effective capillary radius at a specified liquid saturation for a rock with ultra-low permeability.

If initial estimates are obtained from stability analysis for the bulk case and from the modified stability analysis for the capillary pressure case, the convergence behavior and the number of iterations are similar. Figure 7 shows an example for system $\mathrm{V}$ at a specified pressure and temperature. The convergence is clearly quadratic with the same number of 
iterations to reach the solution. Table 5 show the values of the vapor fraction at each iteration and the values of the effective capillary radius for the saturation dependent case.

Table 1: Number of iterations at different $P T$-specifications and capillary radii for system II. Numbers inside parentheses are successive substitution steps and outside parentheses Newton steps. $\mathrm{SF}=$ single phase, $\mathrm{s}=$ Initial estimate from stability analysis. $\left(\right.$ tolerance $\left.=10^{-10}\right)$

\begin{tabular}{cc|llll|lr}
\hline$T(K)$ & $P_{l}($ bar $)$ & bulk & $10(\mathrm{~nm})$ & $5(\mathrm{~nm})$ & $2(\mathrm{~nm})$ & $r_{c}(S) \rightarrow$ & $(\mathrm{nm})$ \\
\hline 150 & 1 & $2(3)$ & $\mathrm{SF}$ & $\mathrm{SF}$ & $\mathrm{SF}$ & $\mathrm{SF}$ & 16.58 \\
200 & 10 & $2(3)$ & $5(3)$ & $5(4)$ & $6(4)$ & $6(3)$ & 3.02 \\
200 & 50 & $6(3) \mathrm{s}$ & $4(3)$ & $4(3)$ & $4(3)$ & $5(3)$ & 3.08 \\
225 & 10 & $2(3)$ & $6(3)$ & $6(3)$ & $6(4)$ & $5(3)$ & 3.02 \\
225 & 50 & $3(3) \mathrm{s}$ & $3(3)$ & $3(3)$ & $4(3)$ & $5(3)$ & 3.02 \\
225 & 75 & $4(3) \mathrm{s}$ & $5(3)$ & $4(3)$ & $3(3)$ & $3(3)$ & 3.02 \\
250 & 1 & $\mathrm{SF}$ & $5(5)$ & $4(5)$ & $6(5)$ & $5(5)$ & 3.02 \\
250 & 10 & $\mathrm{SF}$ & $4(3)$ & $5(3)$ & $5(3)$ & $5(3)$ & 3.02 \\
250 & 50 & $2(3) \mathrm{s}$ & $3(3)$ & $3(3)$ & $5(3)$ & $4(3)$ & 3.02 \\
250 & 75 & $\mathrm{SF}$ & $\mathrm{SF}$ & $\mathrm{SF}$ & $\mathrm{SF}$ & $\mathrm{SF}$ & 3.02 \\
\hline
\end{tabular}

Table 2: Number of iterations at different $P T$-specifications and capillary radii for system III. Numbers inside parentheses are successive substitution steps and outside parentheses Newton steps. $\mathrm{SF}=$ single phase, $\mathrm{s}=$ Initial estimate from stability analysis. $\left(\right.$ tolerance $\left.=10^{-10}\right)$

\begin{tabular}{cc|llll|lr}
\hline$T(K)$ & $P_{l}($ bar $)$ & bulk & $10(\mathrm{~nm})$ & $5(\mathrm{~nm})$ & $2(\mathrm{~nm})$ & $r_{c}(S) \rightarrow$ & $(\mathrm{nm})$ \\
\hline 150 & 1 & $3(3)$ & $\mathrm{SF}$ & $\mathrm{SF}$ & $\mathrm{SF}$ & $\mathrm{SF}$ & 16.58 \\
200 & 10 & $3(3)$ & $5(4)$ & $5(4)$ & $9(8)$ & $6(4)$ & 3.27 \\
200 & 50 & $\mathrm{SF}$ & $\mathrm{SF}$ & $\mathrm{SF}$ & $\mathrm{SF}$ & $\mathrm{SF}$ & 16.58 \\
250 & 100 & $8(3) \mathrm{s}$ & $7(3)$ & $6(4)$ & $6(4)$ & $8(3)$ & 5.40 \\
300 & 50 & $3(3)$ & $3(3)$ & $3(4)$ & $4(4)$ & $5(3)$ & 3.02 \\
300 & 120 & $5(3) \mathrm{s}$ & $5(3)$ & $5(3)$ & $5(3)$ & $6(3)$ & 3.02 \\
350 & 10 & $2(3)$ & $4(4)$ & $5(4)$ & $5(6)$ & $6(4)$ & 3.02 \\
350 & 100 & $5(3)$ & $4(3)$ & $4(3)$ & $5(3)$ & $6(4)$ & 3.02 \\
380 & 10 & $3(3)$ & $5(3)$ & $6(3)$ & $7(3)$ & $8(5)$ & 3.02 \\
\hline
\end{tabular}


Table 3: Number of iterations at different $P T$-specifications and capillary radii for system IV. Numbers inside parentheses are successive substitution steps and outside parentheses Newton steps. $\mathrm{SF}=$ single phase, $\mathrm{s}=$ Initial estimate from stability analysis. $\left(\right.$ tolerance $\left.=10^{-10}\right)$

\begin{tabular}{cc|llll|lr}
\hline$T(K)$ & $P_{l}($ bar $)$ & bulk & $10(\mathrm{~nm})$ & $5(\mathrm{~nm})$ & $2(\mathrm{~nm})$ & $r_{c}(S) \rightarrow$ & \multicolumn{1}{c}{$\mathrm{nm})$} \\
\hline 150 & 1 & $1(3)$ & $\mathrm{SF}$ & $\mathrm{SF}$ & $\mathrm{SF}$ & $\mathrm{SF}$ & 16.58 \\
200 & 1 & $1(3)$ & $4(3)$ & $6(3)$ & $\mathrm{SF}$ & $8(3)$ & 4.38 \\
200 & 50 & $\mathrm{SF}$ & $\mathrm{SF}$ & $\mathrm{SF}$ & $\mathrm{SF}$ & $\mathrm{SF}$ & 16.58 \\
300 & 10 & $3(1)$ & $4(3)$ & $4(3)$ & $8(3)$ & $6(3)$ & 3.02 \\
300 & 100 & $3(3)$ & $3(3)$ & $3(3)$ & $5(3)$ & $4(3)$ & 3.08 \\
300 & 150 & $7(3) \mathrm{s}$ & $5(3)$ & $5(3)$ & $5(3)$ & $5(3)$ & 4.61 \\
400 & 10 & $1(3)$ & $4(3)$ & $4(3)$ & $7(3)$ & $5(3)$ & 3.02 \\
400 & 150 & $8(3) \mathrm{s}$ & $4(3)$ & $4(3)$ & $5(3)$ & $4(3)$ & 3.02 \\
500 & 10 & $1(3)$ & $4(3)$ & $5(3)$ & $5(3)$ & $5(3)$ & 3.02 \\
500 & 150 & $7(3) \mathrm{s}$ & $4(3)$ & $4(3)$ & $4(4)$ & $5(3)$ & 3.02 \\
\hline
\end{tabular}

Table 4: Number of iterations at different $P T$-specifications and capillary radii for system V. Numbers inside parentheses are successive substitution steps and outside parentheses Newton steps. $\mathrm{SF}=$ single phase, $\mathrm{s}=$ Initial estimate from stability analysis. $\left(\right.$ tolerance $=10^{-10}$ )

\begin{tabular}{cc|llll|lr}
\hline$T(K)$ & $P_{l}$ (bar) & bulk & $10(\mathrm{~nm})$ & $5(\mathrm{~nm})$ & $2(\mathrm{~nm})$ & $r_{c}(\mathrm{~S}) \rightarrow$ & $(\mathrm{nm})$ \\
\hline 200 & 10 & $2(3)$ & $\mathrm{SF}$ & $\mathrm{SF}$ & $\mathrm{SF}$ & $\mathrm{SF}$ & 16.58 \\
300 & 10 & $2(3)$ & $4(3)$ & $5(3)$ & $\mathrm{SF}$ & $6(3)$ & 10.61 \\
400 & 10 & $2(3)$ & $3(3)$ & $4(3)$ & $5(4)$ & $6(3)$ & 7.62 \\
400 & 100 & $3(3)$ & $4(3)$ & $\mathrm{SF}$ & $\mathrm{SF}$ & $6(3)$ & 14.67 \\
500 & 10 & $2(3)$ & $4(3)$ & $5(3)$ & $6(3)$ & $6(4)$ & 5.22 \\
500 & 100 & $4(3)$ & $4(3)$ & $5(3)$ & $\mathrm{SF}$ & $5(3)$ & 12.37 \\
600 & 10 & $2(3)$ & $5(3)$ & $6(3)$ & $4(4)$ & $7(3)$ & 3.02 \\
600 & 100 & $4(3)$ & $5(3)$ & $5(3)$ & $6(6)$ & $5(3)$ & 10.31 \\
700 & 50 & $4(3)$ & $5(3)$ & $7(4)$ & $6(2)$ & $5(3)$ & 3.02 \\
750 & 50 & $\mathrm{SF}$ & $\mathrm{SF}$ & $\mathrm{SF}$ & $\mathrm{SF}$ & $\mathrm{SF}$ & 3.02 \\
\hline
\end{tabular}

Table 5: Values of $\beta$ at each iteration and capillary pressure at the solution for the example in Figure 7

\begin{tabular}{c|cccc|ccc}
\hline iteration & $\beta_{\text {bulk }}$ & $\beta_{r_{c}=10}$ & $\beta_{r c=5}$ & $\beta_{r c=2}$ & $\beta_{r_{c}(S)} \rightarrow$ & $r_{c}(S)(\mathrm{nm})$ \\
\hline 1 & 0.3897440 & 0.3107606 & 0.2450603 & 0.0753131 & 0.2450603 & 8.6190022 \\
2 & 0.4279117 & 0.3112307 & 0.2356720 & 0.0723429 & 0.2974333 & 7.7255903 \\
3 & 0.4243932 & 0.3152221 & 0.2400675 & 0.0730090 & 0.2926242 & 7.8109360 \\
4 & 0.4241739 & 0.3153344 & 0.2402755 & 0.0730130 & 0.2924600 & 7.8132762 \\
5 & 0.4241734 & 0.3153346 & 0.2402761 & 0.0730130 & 0.2924599 & 7.8132781 \\
6 & 0.4241734 & 0.3153346 & 0.2402761 & - & 0.2924599 & 7.8132781 \\
\hline$P_{c}$ (bar) & 0 & 23.42659 & 38.43796 & 62.14417 & 28.23014 & - \\
\hline
\end{tabular}




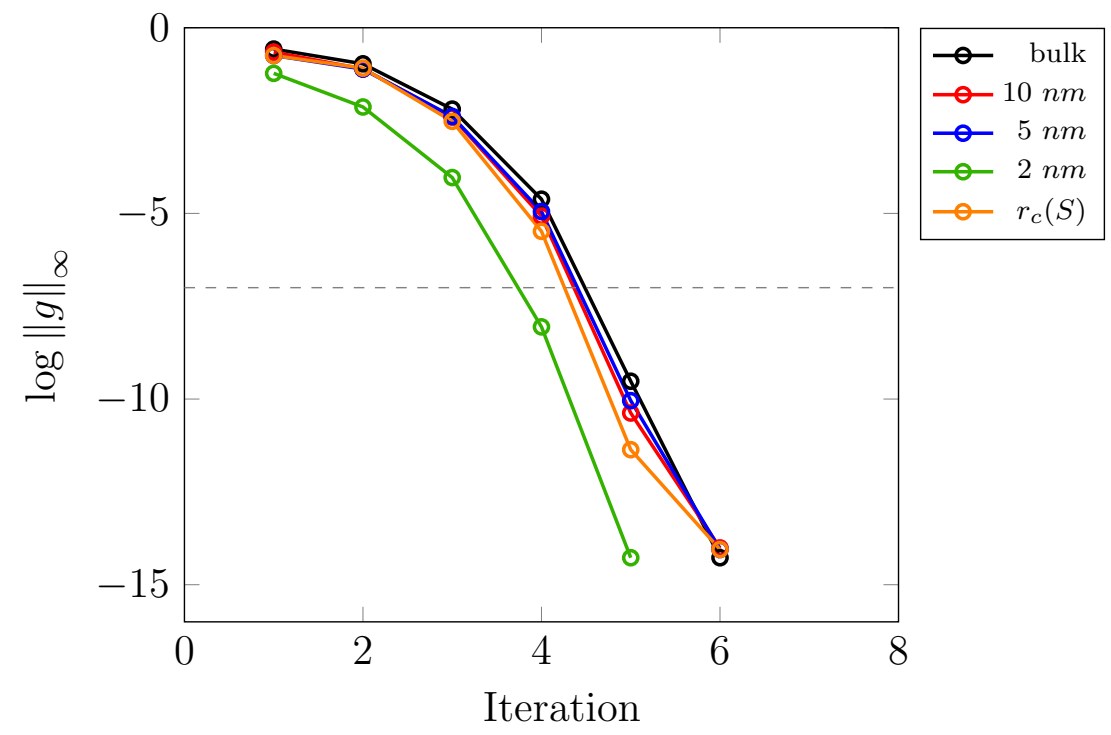

Figure 7: Convergence plot of the gradient at different capillary radii using system V. $T_{\text {spec }}=$ $450 \mathrm{~K}, P_{\text {spec }}=30$ bar

\section{Conclusions}

We present in this paper how to solve two fundamental equilibrium calculation problems, namely, phase envelope construction and flash calculation, in the presence of capillary pressure by use of $V T$-based thermodynamics. $V T$-based thermodynamics has been previously used in various phase equilibrium calculations for bulk phases. Compared with the classical $P T$-based thermodynamics, it avoids repeated solution of phase densities and can easily handle negative pressure values. For calculations involving capillary pressures, it provides the additional advantage that most capillary pressure expressions are functions of molar volumes (e.g. in parachor models) and volume fractions (e.g. in the Leveret $\mathrm{J}$ function used to account for pore size distribution) and it is much simpler to obtain the corresponding derivatives required in the second-order methods. The explicit dependence of interfacial tension and liquid saturation on phase volumes also makes the implementation easier.

Our phase envelope construction algorithm is a direct extension of Michelsen's VT-based phase envelope code. Compared with the PT-based algorithm, the VT-based code uses 
the logarithm of phase molar volumes instead of the logarithm of pressure as independent variables. This requires one more equation for equality of phase pressures in the phase envelope construction for bulk phase. However, the introduction of capillary pressure does not require an additional equation since a modification of the pressure equality equation is sufficient. In comparison with PT-based phase envelope code with capillary pressure, the number of equations is the same.

The VT-based flash involving capillary pressure has a similar form to the $V T$-based flash for bulk phase (Michelsen, 1999). The only modification is to introduce capillary pressure in the original pressure equality equations. This has unfortunately changed the problem from a minimization problem to an equation solving one. The developed VT-based flash is a second order method, and requires several rounds of successive substitution before it. The successive substitution can be initialized by either the Wilson correlation or, more rigorously, a modified stability analysis.

Three fluids (gas, gas condensate, and black oil) are used to test the developed phase envelope and flash algorithms. A capillary radius down to $2 \mathrm{~nm}$ is used to test the algorithms at extreme capillary pressure conditions. For the phase envelope code, the $V T$-based code has given the same features in phase envelope shift as we obtained earlier with a PT-based code. The $V T$-based code turns out to be particularly robust, having no problems with large negative pressure values and showing second order convergence even at a capillary pressure of several hundred bars. For the VT-based flash, the results show that more successive substitution iterations are preferred when capillary pressure is considered, but the second order iterations are nearly the same. Fast second order convergence can be obtained at different capillary radii and also for the case considering pore size distribution.

\section{Supporting Information}

The Supporting Information is available free of charge on the ACS Publication website. 
- System EoS Model Parameters

- Phase Envelope Construction Flowchart

\section{Acknowledgments}

We would like to acknowledge ConocoPhillips and ExxonMobil for their financial on the COMPLEX project. We are also grateful to the Danish Hydrocarbon Research and Technology Centre for partly sponsoring this work.

\section{References}

(1) Heidemann, R. A.; Khalil, A. M. The Calculation of Critical Points. AIChE J. 1980, 26, 769-779.

(2) Nagarajan, N.; Cullick, A.; Griewank, A. New Strategy for Phase Equilibrium and Critical Point Calculations by Thermodynamic Energy Analysis. Part I. Stability Analysis and Flash. Fluid Phase Equilib. 1991, 62, 191-210.

(3) Nagarajan, N. R.; Cullick, A. S.; Griewank, A. New Strategy for Phase Equilibrium and Critical Point Calculations by Thermodynamic Energy Analysis. Part II. Critical Point Calculations. Fluid Phase Equilib. 1991, 62, 211-223.

(4) Michelsen, M. L. State Function Based Flash Specifications. Fluid Phase Equilib. 1999, $158-160,617-626$.

(5) Nichita, D. V.; De-Hemptinne, J.-C.; Gomez, S. Isochoric Phase Stability Testing for Hydrocarbon Mixtures. Pet. Sci. Technol. 2009, 27, 2177-2191.

(6) Mikyska, J.; Firoozabadi, A. A New Thermodynamic Function for Phase-Splitting at Constant Temperature, Moles, and Volume. Am. Inst. Chem. Eng. J. 2011, 47, 18971904. 
(7) Jindrová, T.; Mikyška, J. Fast and Robust Algorithm for Calculation of Two-phase Equilibria at Given Volume, Temperature, and Moles. Fluid Phase Equilib. 2013, 353, $101-114$.

(8) Castier, M. Helmholtz Function-based Global Phase Stability Test and its Link to the Isothermal-Isochoric Flash Problem. Fluid Phase Equilib. 2014, 379, 104-111.

(9) Jindrová, T.; Mikyška, J. General Algorithm for Multiphase Equilibria Calculation at Given Volume, Temperature, and Moles. Fluid Phase Equilib. 2015, 393, 7-24.

(10) Nichita, D. V. Fast and Robust Phase Stability Testing at Isothermal-Isochoric Conditions. Fluid Phase Equilib. 2017, 447, 107-124.

(11) Halldórsson, S.; Stenby, E. H. Isothermal Gravitational Segregation: Algorithms and Specifications. Fluid Phase Equilib. 2000, 175, 175-183.

(12) Pereira, F. E.; Galindo, A.; Jackson, G.; Adjiman, C. S. On the impact of using volume as an independent variable for the solution of P-T fluid-phase equilibrium with equations of state. Comput. Chem. Eng. 2014, 71, 67-76.

(13) Paterson, D.; Michelsen, M. L.; Stenby, E. H.; Yan, W. New Formulations for Isothermal Multiphase Flash (SPE-182706-PA). SPE J. 2017, 20-22.

(14) White, W. B.; Johnson, S. M.; Dantzig, G. B. Chemical Equilibrium in Complex Mixtures. J. Chem. Phys. 1958, 28, 751-755.

(15) Paterson, D.; Michelsen, M.; Stenby, E.; Yan, W. Compositional Reservoir Simulation Using (T, V) Variables-Based Flash Calculation. ECMOR XVI-16th European Conference on the Mathematics of Oil Recovery. 2018.

(16) Brusilovsky, A. I. Mathematical Simulation of Phase Behavior of Natural Multicomponent Systems at High Pressures With an Equation of State. SPE 1992, February, $117-122$. 
(17) Shapiro, A. A.; Stenby, E. H. Thermodynamics of the Multicomponent Vapor-Liquid Equilibrium under Capillary Pressure Difference. Elsevier Sci. B.V. 2001, 178, 17-32.

(18) Sandoval, D. R.; Yan, W.; Michelsen, M. L.; Stenby, E. H. The Phase Envelope of Multicomponent Mixtures in the Presence of a Capillary Pressure Difference. Ind. Eng. Chem. Res. 2016, acs.iecr.6b00972.

(19) Dong, X.; Liu, H.; Hou, J.; Wu, K.; Chen, Z. Phase Equilibria of Confined Fluids in Nanopores of Tight and Shale Rocks Considering the Effect of Capillary Pressure and Adsorption Film. Ind. Eng. Chem. Res. 2016, 55, 798-811.

(20) Fisher, L. R.; Israelachvili, J. N. Experimental Studies on the Applicability of the Kelvin Equation to Highly Curved Concave Menisci. J. Colloid Interface Sci. 1981, $80,528-541$.

(21) Zhong, J.; Riordon, J.; Zandavi, S. H.; Xu, Y.; Persad, A. H.; Mostowfi, F.; Sinton, D. Capillary Condensation in $8 \mathrm{~nm}$ Deep Channels. J. Phys. Chem. Lett. 2018, 9, 497-503.

(22) Yang, Q.; Jin, B.; Banerjee, D.; Nasrabadi, H. Direct Visualization and Molecular Simulation of Dewpoint Pressure of a Confined Fluid in sub-10 nm Slit Pores. Fuel 2019, 235, 1216-1223.

(23) Thommes, M. Physical Adsorption Characterization of Nanoporous Materials. ChemieIngenieur-Technik 2010, 82, 1059-1073.

(24) Shapiro, A.; Stenby, E. Kelvin Equation for a Non-Ideal Multicomponent Mixture. Fluid Phase Equilib. 1997, 134, 87-101.

(25) Luo, S.; Lutkenhaus, J. L.; Nasrabadi, H. Use of Differential Scanning Calorimetry to Study Phase Behavior of Hydrocarbon Mixtures in Nano-Scale Porous Media. J. Pet. Sci. Eng. 2016, 1-8. 
(26) Wang, L.; Parsa, E.; Gao, Y.; Ok, J. T. Experimental Study and Modeling of the Effect of Nanoconfinement on Hydrocarbon Phase Behavior in Unconventional Reservoirs (SPE-169581-MS). SPE West. North Am. Rocky Mt. Jt. Meet. Denver, Colorado, USA, 2014 .

(27) Al, M.; Nasrabadi, H.; Banerjee, D. Fluid Phase Equilibria Experimental Investigation of Confinement Effect on Phase Behavior of Hexane, Heptane and Octane Using Labon-a-Chip Technology. Fluid Phase Equilib. 2016, 423, 25-33.

(28) Travalloni, L.; Castier, M.; Tavares, F. W.; Sandler, S. I. Thermodynamic Modeling of Confined Fluids Using an Extension of the Generalized van der Waals Theory. Chem. Eng. Sci. 2010, 65, 3088-3099.

(29) Shapiro, A.; Stenby, E. Multicomponent Adsorption: Principles and Models. Adsorpt. Theory, Model. Anal. 2002, 375-431.

(30) Rangarajan, B.; Lira, C. T.; Subramanian, R. Simplified local density model for adsorption over large pressure ranges. AIChE J. 1995, 41, 838-845.

(31) Kunz, O.; Klimeck, R.; Wagner, W.; Jaeschke, M. Gerg Tm15; 2007.

(32) Nichita, D. V. Volume-based phase stability testing at pressure and temperature specifications. Fluid Phase Equilib. 2018, 458, 123-141.

(33) Sugden, S. A Relation Between Surface Tension, Density, and Chemical Composition. J. Chem. Soc., Trans. 1924, 125, 1177-1189.

(34) Michelsen, M. Calculation of Phase Envelopes and Critical Points for Multicomponent Mixtures. Fluid Phase Equilib. 1980, 4, 1-10.

(35) Michelsen, M.; Mollerup, J. Thermodynamic Models; Fundamentals \& Computational aspects; 2007. 
(36) Nichita, D. V. A volume-based approach to phase equilibrium calculations at pressure and temperature specifications. Fluid Phase Equilib. 2018, 461, 70-83.

(37) Hebden, M. D. An Algorithm for Minimization Using Exact Second Derivatives; 1973.

(38) McSweeney, T. Modified Cholesky Decomposition and Applications. Ph.D. thesis, The University of Manchester, UK, 2017.

(39) Kou, J.; Sun, S. A Stable Algorithm for Calculating Phase Equilibria with Capillarity at Specified Moles, Colume and Temperature Using a Dynamic Model. Fluid Phase Equilib. 2018, 456, 7-24.

(40) Sherafati, M.; Jessen, K. Stability Analysis for Multicomponent Mixtures Including Capillary Pressure. Fluid Phase Equilib. 2017, 433, 56-66.

(41) Nichita, D. V. Density-based phase envelope construction. Fluid Phase Equilib. 2018, $478,100-113$.

(42) Robinson, D. B.; Peng, D.-Y. The Characterization of the Heptanes and Heavier Fractions for the GPA-Peng-Robinson Programs; RR-28; Gas Processor Association, 1978.

(43) Whitson, C. H.; Sunjerga, S. SPE 155499 PVT in Liquid-Rich Shale Reservoirs. SPE Annu. Tech. Conf. Exhib. San Antonio, Texas, 2012; pp 8-10.

(44) Orangi, A.; Nagarajan, N.; Honarpour, M. M.; Rosenzweig, J. Unconventional Shale Oil and Gas-Condensate Reservoir Production, Impact of Rock, Fluid, and Hydraulic Fractures (SPE 140536). Hydraul. Fract. Technol. Conf. Exhib. The Woodlands, Texas, USA, 2011; pp 1-15.

(45) Brooks, R.; Corey, A. Hydraulic Properties of Porous Media; Colorado State University Hydrology Papers; Colorado State University, 1964. 


\section{Graphical TOC Entry}

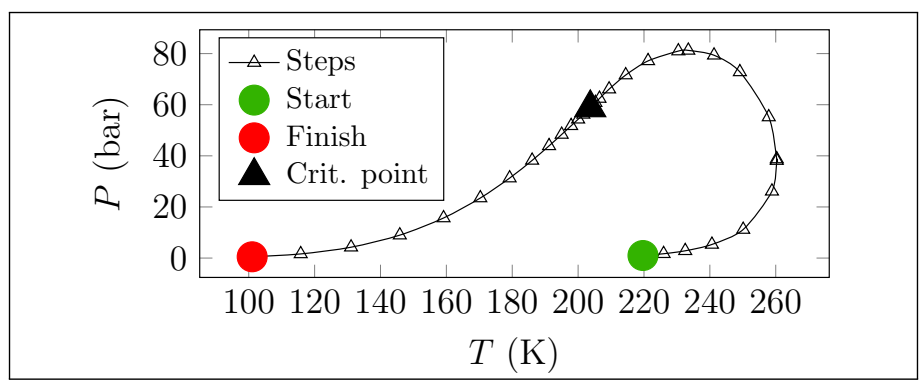

